\title{
CHUYỂN GIAO ĐÀO TẠO - TẦM QUAN TRỌNG CỦA MÔI TRUỪ̛NG LÀM VIỆC TRONG CÁC TỔ CHỨC TẠI THÀNH PHỐ HỒ CHÍ MINH
}

Ngày nhận bài: 25/10/2015

Ngày nhận lại: 18/11/2015

Ngày duyệt đăng: 18/04/2016

\author{
Vũ Việt Hằng ${ }^{1}$ \\ Huỳnh Đạt Lâm
}

\section{TÓM TẮT}

Với mục tiêu tìm hiểu mức độ tác động của các yếu tố thuộc môi trương làm việc đến việc áp dụng nhũng kiến thức, kỹ năng, hành vi và thái độ đã được đào tạo vào công việc của nhân viên (chuyển giao đào tạo) trong các tổ chức trên địa bàn Thành phố Hồ Chí Minh, các tác giả của đề tài "Chuyển giao đào tạo - Tầm quan trọng của môi truờng làm việc" đã khảo sát ý kiến của 178 nhân viên được công ty cư đi đào tạo tù 3 đến 24 tháng. Thông qua nghiên cứu thực nghiệm kết hợp hai phwơng pháp co bản: nghiên cưu định tính (thảo luận nhóm và phỏng vấn sâu) cùng nghiên cưu định lượng (dùng bảng câu hỏi khảo sát), các tác giả đã tìm thấy 4 yếu tố có ý nghĩa thống kê ảnh hưởng tích cực đến chuyển giao đào tạo gồm: Co hội áp dụng, Sụ hỗ trợ của đồng nghiệp, Sụ hố trợ của cấp trên và Sụ sẵn sàng thay đổi.

Tù khóa: Đào tạo; Chuyển giao đào tạo; Môi truò̀ng làm việc.

Transfer of training - the importance of work environment in organisations in Ho Chi Minh City

\section{ABSTRACT}

The Aim of the study is to find out what factors of work environment are that affect the extent to which trainees apply the knowledge, skills, behaviors, and attitudes gained in training to their jobs (transfer of training) in Ho Chi Minh City. The research "Transfer of training - The importance of work environment" collects data from 178 employees who were trained by the company from 3 to 24 months. By the survey research that combine two methods: qualitative (group discussion and in depth interview) and quantitative (questionnaire) the authors found 4 factors that significantly and positively related to transfer of training: opportunity to use, peer support, manager support and openness to change.

Keywords: Training; Transfer of training; Work environment.

\section{Giới thiệu}

Đối mặt với nền kinh tế luôn biến đổi, toàn cầu hóa và cạnh tranh, hầu như công ty nào cũng đầu tư vào lĩnh vực đào tạo cho nhân viên và hy vọng rằng khoản đầu tư này sẽ tạo ra hiệu quả làm việc cũng như nâng cao lợi thế cạnh tranh cho công ty (Yamnill và McLean,
2001). Việc đảm bảo cho nhân viên có đầy đủ kỹ năng cũng như kiến thức để thực hiện các công việc hiện tại và tương lai là một thách thức lớn nhất mà các công ty đang đối mặt. Đào tạo nhân viên là một phần không thể thiếu trong bất cứ công ty nào, nhưng đào tạo chỉ có thể được gọi là có hiệu quả khi kết quả

\footnotetext{
${ }^{1}$ TS, Trường Đại học Mở TP.HCM. Email: hang.vv@ou.edu.vn

${ }^{2}$ Công ty SCG Trading Việt Nam. Email: hdatlam@outlook.com
} 
đào tạo được áp dụng vào công việc. Nếu nhân viên được đào tạo có khả năng ứng dụng các kiến thức và kỹ năng đã học làm tăng kết quả công việc thì việc cử nhân viên đi đào tạo được cho là thành công. Chuyển giao đào tạo là mức độ nhân viên ứng dụng những kiến thức, kỹ năng và hành vi thu nhận được từ đào tạo một cách có hiệu quả vào môi trường làm việc (Baldwin và Ford, 1988). Theo hiệp hội đào tạo và phát triển Hoa Kỳ ASTD (2012), riêng tại nước Mỹ thì các công ty chi 164,2 tỉ \$ cho việc đào tạo nhân viên nhưng chưa tới $20 \%$ kiến thức và kỹ năng đào tạo chuyển giao được thể hiện trong quá trình thực hiện công việc. Qua đó, có thể nhận ra rằng phần lớn nội dung trong chương trình đào tạo nhân viên đã không được chuyển giao vào công việc.

Tại Việt Nam, nghiên cứu về chuyển giao đào tạo còn rất mới và hầu như chưa có công trình nào đề cập đến vấn đề này. Hiện tại, các nhà nghiên cứu về đào tạo và các chuyên gia quản lý nhân sự hầu như chỉ tập trung vào tìm hiểu các vấn đề như: thiết kế, phát triển, đánh giá hiệu quả của các chương trình đào tạo mà ít chú tâm đến vấn đề chuyển giao đào tạo. Đặc biệt là những nghiên cứu về ảnh hưởng của môi trường làm việc đến chuyển giao đào tạo còn khá hiếm hoi.

Nghiên cứu Foxon (1993) cho rằng trong các yếu tố cản trở đến chuyển giao đào tạo thì yếu tố môi trường làm việc có ảnh hưởng mạnh nhất (chiếm 42\%). Nghiên cứu các yếu tố của môi trường làm việc tác động lên chuyển giao đào tạo sẽ góp phần giúp các chuyên gia thuộc lĩnh vực nhân sự, các chuyên gia đào tạo có cái nhìn mới mẻ và sâu hơn về vấn đề này, từ đó có các biện pháp để làm tăng hiệu quả của các chương trình đào tạo, duy trì năng lực cho nhân viên, tạo lợi thế cạnh tranh nhất định cho tổ chức kinh doanh.

\section{Cơ sở lý thuyết và mô hình nghiên cứu}

\subsection{Các khái niệm}

Đào tạo là những cố gắng của tổ chức nhằm thay đổi kiến thức, kỹ năng, hành vi và thái độ của nhân viên để đáp ứng các yêu cầu về hiệu quả của công việc. Nói cách khác, Đào tạo là hành động nhằm cải thiện kiến thức và kỹ năng của nhân viên để thực hiện những công việc cụ thể (Edwin B Flippo, 1984).

Chuyển giao đào tạo là mức độ nhân viên ứng dụng một cách có hiệu quả những gì được đào tạo vào môi trường làm việc (Balwin \& Ford, 1988).

Môi trường làm việc là tất cả các điều kiện mà trong đó nhân viên thực hiện những công việc hay nhiệm vụ thuộc về trách nhiệm của họ (Gielen, 1996).

\subsection{Co' sở lý thuyết}

Lý thuyết chuyển giao đào tạo của Kirkpatrick (1998): Theo lý thuyết này kết quả đào tạo được đánh giá theo bốn cấp bao gồm: Phản hồi, học hỏi, hành vi công việc và kết quả công việc, cụ thể là:

$>$ 1. Phản hồi: nhân viên được đào tạo cảm thấy thế nào về nội dung và hình thức học

2. Học hỏi: nhân viên đã được học những gì

$>$ 3. Hành vi công việc: những gì đã được đào tạo có được chuyển giao vào công việc hay không

> 4. Kết quả: việc cử nhân viên đi đào tạo ảnh hưởng đến doanh thu, lợi nhuận của doanh nghiệp như thế nào?

\subsection{Nghiên cúu liên quan}

Nghiên cứu của Baldwin \& Ford (1988) chỉ ra rằng, để chuyển giao đào tạo đạt được thì nhân viên được đào tạo phải khái quát hóa những gì được đào tạo vào môi trường công việc và duy trì trong một thời gian. Các yếu tố đầu vào của đào tạo bao gồm đặc điểm học viên, thiết kế chương trình đào tạo và môi trường làm việc.

Foxon (1993) nghiên cứu về ảnh hưởng của động cơ và sự hỗ trợ cấp trên đến chuyển giao đào tạo. Khi đưa ra tiến trình của sự chuyển giao đào tạo, ông lập luận rằng: chuyển giao đào tạo không phải là hiện tượng đơn lẻ mà là một quá trình gồm hai mức độ: mức độ "chấp nhận" và mức độ "tối ưu hóa" chuyển giao đào tạo. Tiến trình chuyển giao đào tạo cho biết người được đào tạo cố gắng sử dụng những kiến thức và kỹ năng mới hay không sử dụng chúng (tức là chuyển giao đào tạo thất bại). Chuyển giao đào tạo sẽ khó đạt 
được nếu những người được đào tạo không đi theo trình tự các giai đoạn như sau: có ý định chuyển giao, bắt đầu chuyển giao, chuyển giao một phần, duy trì chuyển giao có ý thức và cuối cùng là chuyển giao đào tạo một cách vô thức. Khi được cử đi đào tạo, tất cả nhân viên đều được kỳ vọng là sẽ áp dụng được kiến thức, kỹ năng đào tạo vào trong công việc. Nếu nhân viên không duy trì, không áp dụng hay quên đi những gì đã được đào tạo thì chuyển giao đào tạo coi như thất bại. Foxon chỉ ra năm yếu tố lớn nhất cản trở đến chuyển giao đào tạo, đó là:

$>$ Yếu tố môi trường làm việc (chiếm 42\%)

$>$ Yếu tố chương trình đào tạo (chiếm $22 \%$ )

$>$ Yếu tố đặc điểm cá nhân (chiếm 21\%)

$>$ Yếu tố động cơ của nhân viên (chiếm $13 \%$ )

> Phương pháp đào tạo (chiếm 13\%)

Mô hình nghiên cứu của Holton và cộng sự (2000) về hệ thống chuyển giao đào tạo (Learning Transfer System Inventory), đưa ra 16 yếu tố được đo lường bởi 68 biến quan sát có tác động tích cực hay tiêu cực đến chuyển giao đào tạo. Mô hình này đã được các nhà nghiên cứu khác kế thừa và áp dụng sau này: tại Đài Loan là nghiên cứu của Chen (2003), Yamnill (2001) đã áp dụng mô hình LTSI thành công tại Thái Lan, Devos và cộng sự (2007) tại Pháp.

\subsection{Mô hình nghiên cứu đề xuất}

Mô hình nghiên cứu bao gồm một biến phụ thuộc và 4 biến độc lập được đề xuất sau khi tìm hiểu kỹ các nghiên cứu trước. Trong đó, 4 biến độc lập là: Cơ hội áp dụng, sự Hỗ trợ của đồng nghiệp, sự Hỗ trợ của cấp trên và sự Sẵn lòng thay đổi; biến phụ thuộc là Chuyển giao đào tạo. Năm yếu tố này được đo lường bởi 29 biến quan sát, sử dụng thang đo của Holton và cộng sự đã được hiệu chỉnh qua nghiên cứu định tính. Cụ thể là, thông qua thảo luận nhóm, các tác giả đã bổ sung được 2 biến quan sát vào thang đo biến độc lập "Cơ hội áp dụng"; 1 biến quan sát vào thang đo biến độc lập "Sự hỗ trợ của đồng nghiệp" và 4 biến quan sát vào thang đo biến phụ thuộc "Chuyển giao đào tạo". Nhờ vậy, hệ thống thang đo trong nghiên cứu đã được hiệu chỉnh cho phù hợp hơn với hoàn cảnh thực tế tại Việt Nam.

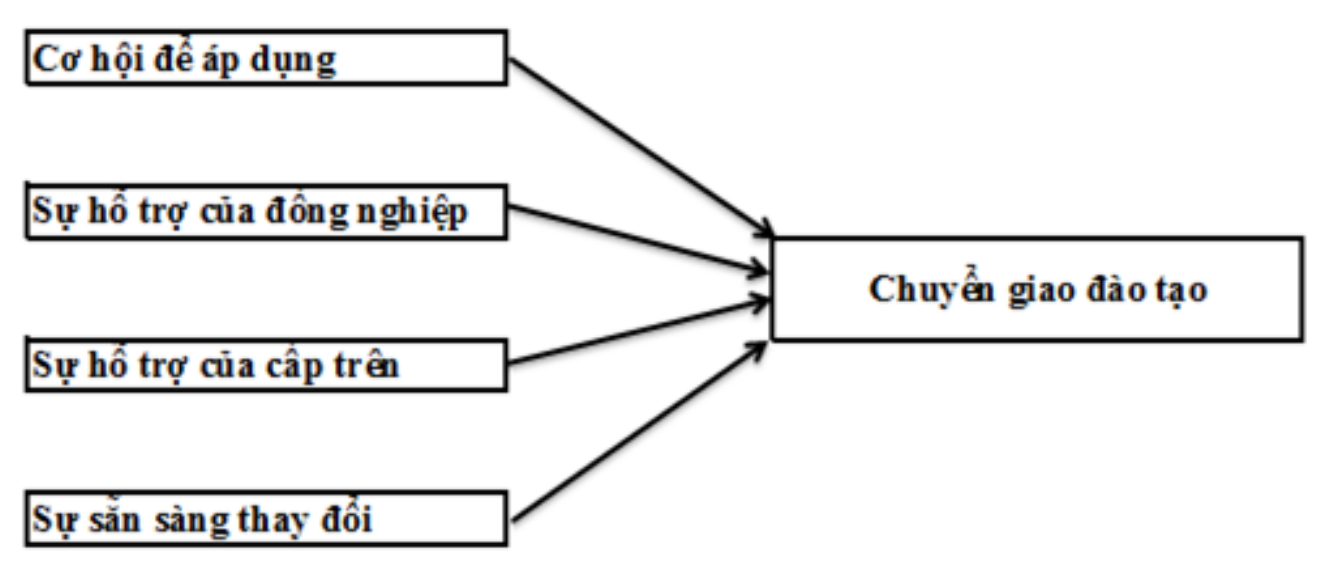

Hình 1. Mô hình nghiên cứu đề xuất

Mối liên hệ giữa Cơ hội để áp dụng và Chuyển giao đào tạo

Để có thể áp dụng được những kiến thức và kỹ năng đã học trong khóa đào tạo của công ty, nhân viên phải có cơ hội. Theo Holton và cộng sự (2000) định nghĩa "cơ hội để áp dụng là mức độ người được đào tạo được cung cấp các nguồn lực cần thiết và được giao các công việc phù hợp cho phép họ áp dụng những kiến thức và kỹ năng thu nhận được vào công việc". Một vài nghiên cứu đã chứng minh được tầm quan trọng của cơ hội để áp dụng tác động đến việc áp dụng kiến thức và kỹ năng trong công việc của nhân viên (Lim và Johnson, 2002). Nếu công việc mà không có cơ hội áp dụng thì mức độ 
chuyển giao đào tạo khó có xảy ra được. Vì vậy cơ hội áp dụng là yếu tố có tác động tích cực đến chuyển giao đào tạo.

Giả thuyết 1: Co họi áp dưng có tác động tích cưc tới chuyển giao đào tạo

$>$ Mối liên hệ giữa Sự hỗ trợ của đồng nghiệp và Chuyển giao đào tạo

Sự hỗ trợ của đồng nghiệp là sự giúp đỡ của đồng nghiệp đối với việc áp dụng kiến thức kỹ năng vào công việc Holton và cộng sự (2000). Nghiên cứu của Facteau và cộng sự (1995); Hawley và Barnard (2005), chỉ ra rằng sự hỗ trợ của đồng nghiệp có tác động tích cực đến chuyển giao đào tạo. Sự hỗ trợ của đồng nghiệp tác động mạnh lên chuyển giao đào tạo bởi khi làm chung với nhau, sự chấp nhận, động viên, khích lệ, hay chí ít là không chế nhạo, cười cợt, xem thường, ...là rất quan trọng.

Giả thuyết 2: Sư hỗ trợ của đồng nghiệp có tác động tích cưc tới chuyển giao đào tạo

> Mối liên hệ giữa Sự hỗ trợ của cấp trên và Chuyển giao đào tạo

Sự hỗ trợ của cấp trên là mức độ cấp trên giúp đõ̃ và khuyến khích việc sử dụng kiến thức và kỹ năng mới vào công việc của nhân viên (Holton và cộng sự, 2000). Theo Baldwin và Ford (1988), cấp trên có thể tạo ra những cơ hội cho nhân viên áp dụng những kiến thức và kỹ năng đã được đào tạo vào công việc. Chiaburu (2005) chứng minh mối quan hệ giữa cấp trên có ảnh hưởng tích cực đến chuyển giao đào tạo. Nếu như trong doanh nghiệp mà cấp trên bảo thủ, thích làm việc theo lề lói thông thường thì thật khó để nhân viên sau được đào tạo về có thể chuyển giao thành công. Vì vậy sự hỗ trợ của cấp trên là một yếu tố không thể thiếu nếu muốn chuyển giao đào tạo xảy ra.

Giả thuyết 3: Sư hỗ trợ của cấp trên có tác động tích cục tới chuyển giao đào tạo

Mối liên hệ giữa Sự sẵn sàng thay đổi và Chuyển giao đào tạo

Sự sẵn sàng thay đổi được định nghĩa là sự sẵn sàng thích nghi và chấp nhận thay đổi (Wanberg và Banas, 2000). Theo Holton và cộng sự (2000) sự sã̃n sàng thay đổi có tác động tích cực đến chuyển giao đào tạo và được định nghĩa là những quan niệm đang chiếm ưu thế trong nhóm làm việc mà việc sử dụng những gì đã được đào tạo vào công việc được khuyến khích. Nếu văn hóa của công ty mà thích làm việc theo cách thức cũ, e ngại cách làm mới, không khuyến khích những cách làm mới thì sự chuyển giao đào tạo khó xảy ra. Đặc biệt qua cuộc thảo luận nhóm với đáp viên thì hầu như đều cho rằng văn hóa chấp nhận và thích nghi với cái mới (sự sã̃n sàng thay đổi) có tác động tích cực đến chuyển giao đào tạo. Họ cũng chỉ ra rằng nếu ai đó làm việc trong một doanh nghiệp nhà nước thì cơ hội chuyển giao đào tạo khó xảy ra vì sự sã̃n sàng thay đổi của nó thấp. Vì vậy đây cũng là một yếu tố khá quan trọng ảnh hưởng đến chuyển giao đào tạo.

Giả thuyết 4: Sư sã̃n lòng thay đổi có tác động tích cưc tới chuyển giao đào tạo

\section{Phương pháp nghiên cứu}

Đề tài sử dụng hai phương pháp nghiên cứu là định tính và định lượng, được tiến hành theo các bước:

> Nghiên cứu định tính sơ bộ: Thảo luận nhóm để khám phá các yếu tố có ảnh hưởng đến chuyển giao đào tạo và xem xét, hiệu chỉnh hay lược bỏ các thang đo và điều chỉnh mô hình (nếu có) để phù hợp với bối cảnh nghiên cứu tại Thành phố Hồ Chí Minh.

> Nghiên cứu định lượng: Tiến hành thống kê mô tả, phân tích độ tin cậy thang đo, phân tích nhân tố khám phá EFA, phân tích tương quan và phân tích hồi quy.

> Nghiên cứu định tính lần 2: Sau khi phân tích kết quả nghiên cứu chính thức từ định lượng sẽ thực hiện nghiên cứu định tính lần 2 bằng hình thức phỏng vấn tay đôi với các chuyên gia để đối chiếu kết quả của nghiên cứu với các ý kiến và nhận định từ họ.

\section{Kết quả nghiên cứu}

\subsection{Mô tả thông tin mẫu}

Mẫu của nghiên cứu này được thu thập theo phương pháp thuận tiện với bảng câu hỏi 
khảo sát giấy và khảo sát trực tuyến qua công cụ https://docs.google.com. Kết quả là nhận được 178 câu trả lời hợp lệ: 84 câu trả lời từ khảo sát trực tuyến và 94 câu trả lời từ khảo sát giấy. Kết quả trả lời được nhập liệu vào phần mềm SPSS 22.
Giới tính

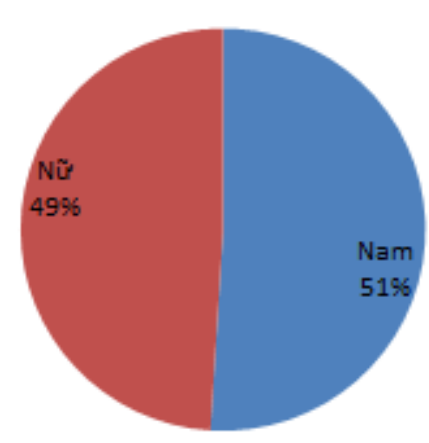

Cấp bậc

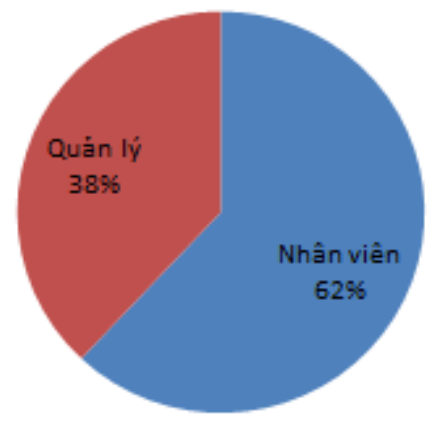

Độ tuổi

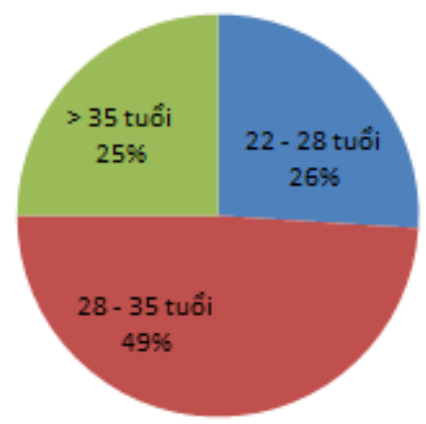

Kinh nghiệm

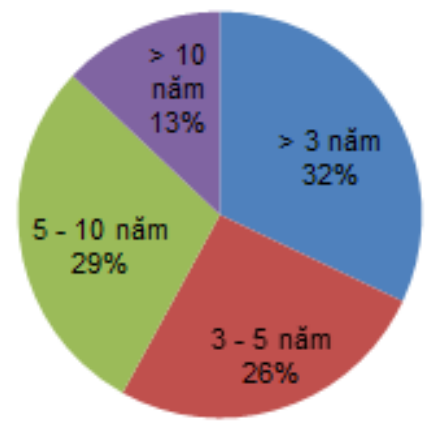

Thu nhập

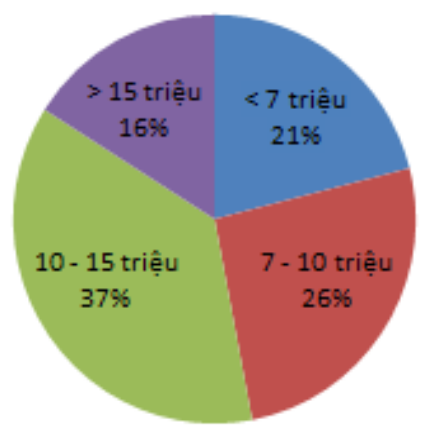

Hình 2. Mô tả mẫu theo các đặc điểm

\subsection{Kết quả thống kê mô tả}

Kết quả thống kê cho thấy các câu trả lời trải dài từ mức thấp đến mức cao ở tất cả các câu hỏi. Điều này có nghĩa cùng một phát biểu nhưng có người đánh giá là "rất không đồng ý", có người đánh giá là "rất đồng ý". Nhìn chung giá trị trung bình của các biến quan sát đều lớn hơn 3 (giá trị trung hòa) ngoại trừ biến CD1 (Đồng nghiệp trong phòng ban của tôi ưa thích sử dụng các kỹ năng và kiến thức cũ hơn là sử dụng các kiến thức và kỹ năng đã được đã được đào tạo vào công việc) bằng 2.88 .

Các biến quan sát phụ thuộc cũng được đánh giá từ mức độ 1 (rất không đồng ý) đến mức độ 5 (rất đồng ý). Giá trị trung bình hầu hết các biến quan sát đều lớn hơn 3 , ngoại trừ thang đo DT6 bằng 2.61 (Sau khi được đào tạo về, tôi luôn tìm cách áp dụng kiến thức và kỹ năng vào trong công việc). Phát biểu DT3 (Kiến thức và kỹ năng đã được đào tạo rất hữu ích để tôi giải quyết công việc hiện tại) được đánh giá từ mức độ 2 (không đồng ý) đến mức độ 5 (rất đồng ý) và có giá trị trung bình cao nhất (3.85).

\section{3. Đánh giá độ tin cậy của thang đo}

7 biến quan sát có hệ số tương quan biến tổng hiệu chỉnh nhỏ hơn 0.3 và hệ số Cronbach's Alpha từ 0.6 trở xuống đã bị loại. Đó là 2 biến quan sát $\mathrm{DN} 2$ và $\mathrm{DN} 5$ trong thang đo "Sự hỗ trợ của đồng nghiệp"; 2 biến quan sát $\mathrm{CD} 1$ và $\mathrm{CD} 5$ trong thang đo "Sự sẵn sàng thay đổi" và ba biến quan sát DT6, DT7 và DT8 trong thang đo "Chuyển giao đào tạo". Như vậy, số biến quan sát được giữ lại là 22 . 
Bảng 1. Kết quả phân tích độ tin cậy của 5 thang đo

\begin{tabular}{|l|l|l|}
\hline \multicolumn{1}{|c|}{ Thang đo } & \multicolumn{1}{|c|}{ Số biến quan sát } & Hệ số Cronbach's Alpha \\
\hline Cơ hội áp dụng & $5(\mathrm{CH} 1, \mathrm{CH} 2, \mathrm{CH} 3, \mathrm{CH} 4, \mathrm{CH} 5)$ & .835 \\
\hline Sự hỗ trợ của đồng nghiệp & $3(\mathrm{DN} 1, \mathrm{DN} 3, \mathrm{DN} 4)$ & .847 \\
\hline Sự hỗ trợ cấp trên & $6(\mathrm{CT} 1, \mathrm{CT} 2, \mathrm{CT} 3, \mathrm{CT} 4, \mathrm{CT} 5, \mathrm{CT} 6)$ & .689 \\
\hline Sự sẵn sàng thay đổi & $3(\mathrm{CD} 2, \mathrm{CD} 3, \mathrm{CD} 4)$ & .8 \\
\hline Chuyển giao đào tạo & $5(\mathrm{DT} 1, \mathrm{DT} 2, \mathrm{DT} 3, \mathrm{DT} 4, \mathrm{DT} 5)$ & .88 \\
\hline Tổng cộng & 22 & \\
\hline
\end{tabular}

\subsection{Phân tích nhân tố khám phá EFA}

Phân tích nhân tố khám phá (EFA) chủ yếu được dùng để thu nhỏ và tóm tắt dữ liệu. Sử dụng phương pháp trích nhân tố Principal Component với phép quay Varimax và điểm dừng khi trích các yếu tố có Eigenvalues lớn hơn hoặc bằng 1 đối với 25 biến quan sát đo lường 5 khái niệm: cơ hội áp dụng, sự hỗ trợ của đồng nghiệp, sự hỗ trợ của cấp trên, sự sẵn sàng thay đổi và chuyển giao đào tạo.

Kết quả phân tích nhân tố khám phá EFA đối với các biến độc lập như sau: Bốn nhân tố được rút trích có $: \mathrm{KMO}=0.712>0.5, \mathrm{Sig}=0$ $<0.05$, Eigenvalue nhỏ nhất tại 1.478 và tổng phương sai trích $73 \%>50 \%$ và tất cả các hệ số tải nhân tố đều lớn hơn 0.6. Điều này chứng tỏ 4 nhân tố trích được đã giải thích $61 \%$ biến thiên của tập dữ liệu.

\section{Bảng 2. Phân tích nhân tố khám phá EFA đối với biến độc lập}

\begin{tabular}{|l|l|l|l|l|}
\hline \multirow{2}{*}{} & \multicolumn{5}{|c|}{1} & \multicolumn{1}{l|}{} \\
\cline { 2 - 5 } & 1 & \multicolumn{1}{l|}{2} & 3 & 4 \\
\hline CH1 & .883 & & & \\
\hline CH3 & .847 & & & \\
\hline CH2 & .775 & & & \\
\hline DN1 & & .910 & & \\
\hline DN4 & & .847 & & \\
\hline DN3 & & .798 & & \\
\hline CT2 & & & .879 & \\
\hline CT1 & & & .839 & \\
\hline CT3 & & & .831 & \\
\hline CD3 & & & & .877 \\
\hline CD4 & & & & .819 \\
\hline CD2 & & & & .775 \\
\hline
\end{tabular}

\subsection{Phân tích tương quan}

Phân tích tương quan nhằm kiểm định mối tương quan tuyến tính chặt chẽ giữa biến phụ thuộc và biến độc lập vì điều kiện để phân tích hồi quy xảy ra là phải tương quan với nhau. Kết quả của phân tích tương quan chỉ ra rằng yếu tố “đồng nghiệp" và "sự sẵn sàng thay đổi” có mối tương quan mạnh nhất đến chuyển giao đào tạo. Mối tương quan giữa yếu tố "sự hỗ trợ của cấp trên" và “chuyển giao đào tạo" hơi yếu (0.226). 
Bảng 3. Kết quả phân tích tương quan

\begin{tabular}{|l|l|l|l|l|l|}
\hline & \multicolumn{1}{|c|}{ CH } & \multicolumn{1}{c|}{ DN } & \multicolumn{1}{c|}{ CT } & \multicolumn{1}{c|}{ CD } & DT \\
\hline CH & 1 & & & & \\
\hline DN & $.266^{* *}$ & 1 & & & \\
\hline CT & -0.036 & 0.05 & 1 & & \\
\hline CD & $.274^{* *}$ & $.338^{* *}$ & 0.082 & 1 & \\
\hline DT & $.318^{* *}$ & $.508^{* *}$ & $.266^{* *}$ & $.412^{* *}$ & 1 \\
\hline
\end{tabular}

4.6. Phân tích hồi quy đa biến

Kết quả phân tích tương quan giữa các biến cho thấy có mối quan hệ tuyến tính giữa các biến độc lập và biến phụ thuộc. Đây là cơ sở để thực hiện phân tích hồi quy.

Bảng 4. Kết quả hồi quy các yếu tố ảnh hưởng đến chuyển giao đào tạo

\begin{tabular}{|l|l|l|l|l|l|l|l|}
\hline \multirow{2}{*}{$\begin{array}{c}\text { Giả } \\
\text { thuyết }\end{array}$} & \multicolumn{2}{|c|}{$\begin{array}{c}\text { Hệ số hồi quy chura } \\
\text { chuẩn hóa }\end{array}$} & $\begin{array}{c}\text { Hệ số hồi } \\
\text { quy chuần } \\
\text { hóa }\end{array}$ & $\mathrm{t}$ & \multicolumn{2}{|c|}{ Sig } & \multicolumn{2}{|c|}{ Đo lường đa cộng tuyến } \\
\cline { 2 - 8 } & $\mathrm{B}$ & $\begin{array}{c}\text { Sai sốn } \\
\text { chuâ̂n }\end{array}$ & Beta & & & $\begin{array}{c}\text { Mức độ chấp } \\
\text { nhận biến }\end{array}$ & $\begin{array}{c}\text { Hệ số phóng } \\
\text { đại phương } \\
\text { sai }\end{array}$ \\
\hline & 0.338 & 0.351 & & .961 & .338 & & \\
\hline $\mathrm{CH}$ & 0.191 & 0.073 & .165 & 2.634 & .009 & .887 & 1.128 \\
\hline $\mathrm{DN}$ & 0.334 & 0.056 & .378 & 5.915 & .000 & .852 & 1.173 \\
\hline $\mathrm{CT}$ & 0.234 & 0.059 & .235 & 3.962 & .000 & .988 & 1.012 \\
\hline $\mathrm{CD}$ & 0.217 & 0.063 & .220 & 3.424 & .001 & .844 & 1.185 \\
\hline
\end{tabular}

Từ kết quả trên, có thể kết luận như sau:

> Hệ số phóng đại phương sai VIF lớn nhất là 1.185 nhỏ hơn 2 nên không có hiện tượng đa cộng tuyến xảy ra.

$>$ Tất cả các giá trị Sig đều nhỏ hơn 0.5 nên mô hình hồi quy phù hợp với tổng thể.

$>R^{2}$ hiệu chỉnh bằng 0.384 . Điều này chứng tỏ bốn biến độc lập (1) Cơ hội áp dụng, (2) Sự hỗ trợ của đồng nghiệp, (3) Sự hỗ trợ của cấp trên và (4) Sự sã̃n sàng thay đổi giải thích được gần $38,4 \%$ sự biến thiên của biến phụ thuộc "Chuyển giao đào tạo". Trong đó, biến Sự hỗ trợ của đồng nghiệp có tác động mạnh nhất đến Chuyển giao đào tạo thông qua hệ số beta chuẩn hóa 0.378 .

Bảng 5. Tóm tắt mô hình hồi quy

\begin{tabular}{|l|c|c|c|c|}
\hline Mô hình & $\mathrm{R}$ & $R^{2}$ & $R^{2}$ hiệu chỉnh & Sai số dự đoán \\
\hline 1 & $.631^{\mathrm{a}}$ & .398 & .384 & .62859 \\
\hline
\end{tabular}


Phương trình hồi quy như sau:

Chuyển giao đào tạo $=0.378^{*}$ Sự hỗ trợ của đồng nghiệp $+0.235^{*}$ Sự hỗ trợ cấp trên $+0.22 *$ Sự sẵn sàng thay đổi $+0.165^{*}$ Cơ hội áp dụng.

Qua kết quả phân tích hồi quy, các giả thuyết đặt ra được chấp nhận. Điều này có nghĩa là nếu môi trường làm việc có nhiều cơ hội áp dụng kiến thức kỹ năng mới, người đào tạo nhận được nhiều sự hỗ trợ của đồng nghiệp và cấp trên cũng như có một sự cởi mở trong áp dụng thì mức độ chuyển giao đào tạo càng cao.

Kết quả kiểm định $\mathrm{T}$-test và Anova cũng cho thấy sự khác biệt về giới tính, cấp bậc, kinh nghiệm, thu nhập và độ tuổi không tạo nên sự khác biệt trong mức độ ảnh hưởng của các biến độc lập đến biến Chuyển giao đào tạo.

\subsection{So sánh kết quả với nghiên cúu trước và Thảo luận với chuyên gia}

Nghiên cứu của Foxon (1993) có nhận định rằng yếu tố môi trường làm việc ảnh hưởng đến mức độ chuyển giao đào tạo là $42 \%$. Nghiên cứu này có nét khá tương đồng là môi trường làm việc cũng ảnh hưởng gần $40 \%$ đến mức độ áp dụng những kiến thức và kỹ năng đã được đào tạo vào công việc hiện tại.

Sau khi có kết quả phân tích định lượng, các tác giả đã tiến hành phân tích định tính (lần 2) thông qua phương pháp phỏng vấn tay đôi (hỏi ý kiến chuyên gia) nhằm làm sáng tỏ thêm kết quả. Khi thảo luận, hai chuyên gia đang làm việc trong lĩnh vực nhân sự đã có một số ý kiến như sau:

> Về đề tài: Ý kiến chung là đề tài nghiên cứu thú vị, thiết thực, nếu có điều kiện thì nên mở rộng thêm sang một số yếu tố có thể tác động đến chuyển giao đào tạo khác như: động lực, đặc điểm cá nhân, chương trình đào tạo...

> Về kết quả nghiên cứu: Căn cứ vào mô hình hồi quy thì biến đồng nghiệp có tác động tích cực nhất đến chuyển giao đào tạo. Tuy nhiên, một chuyên gia có ý kiến phản biện rằng kết quả nghiên cứu này không thật thuyết phục. Theo ông, yếu tố tác động mạnh nhất phải là sự sẵn lòng thay đổi vì nếu làm việc trong một môi trường cứng nhắc, không chịu tiếp thu cái mới thì cho dù nhân viên đó có cố gắng áp dụng những gì đã được đào tạo đến đâu nhưng vì "ngựa quen đường cũ " nên thật khó để chuyển giao đào tạo thành công. Ông cũng cho rằng để có được kết quả chính xác hơn thì nên phân chia đối tượng khảo sát thành hai nhóm: nhân viên văn phòng và nhân viên $k y ̃$ thuật. Nhưng chuyên gia thứ hai lại cho rằng kết quả nghiên cứu phù hợp với thực tế vì đồng nghiệp là những người gần gũi nhất với nhân viên được cử đi đào tạo. Nếu họ ủng hộ, khuyến khích và giúp đỡ thì việc chuyển giao đào tạo sẽ diễn ra thành công hơn.

\section{Kết luận và hàm ý quản trị 5.1. Kết luận}

Môi trường làm việc tác động đến kết quả chuyển giao đào tạo qua bốn yếu tố: cơ hội áp dụng, sự hỗ trợ của đồng nghiệp, sự hỗ trợ của cấp trên và sự sẵn sàng thay đổi. Thông qua phân tích mối tương quan, hồi quy đa biến thì nghiên cứu đã đi tới kết luận rằng bốn biến đều có tác động tích cực đến sự chuyển giao đào tạo. Trong đó, biến đồng nghiệp ảnh hưởng mạnh nhất (beta chuẩn hóa là 0.378), tiếp theo là sự hỗ trợ của cấp trên (beta chuẩn hóa là 0.235 ), kế tiếp là sự sẵn sàng thay đổi (hệ số beta chuẩn hóa 0.22 ) và biến cơ hội áp dụng có mức độ ảnh hưởng thấp nhất $(0,165)$. Ngoài ra, $R^{2}$ hiệu chỉnh bằng $38,4 \%$ chứng tỏ môi trường làm việc có tác động khá lớn đến sự chuyển giao đào tạo. Nếu so với nghiên cứu của Foxon (1993) thì kết quả có sự tương đồng. Phân tích mối liên hệ giữa biến định tính và biến định lượng không cho thấy sự khác nhau giữa biến giới tính, cấp bậc hay chức vụ, độ tuổi, kinh nghiệm làm việc và thu nhập trong mức độ ảnh hưởng đến chuyển giao đào tạo. Điều này chứng tỏ, khi quyết định cử một nhân viên nào đó đi đào tạo thêm thì bộ phận nhân sự không cần quan tâm lắm đến tuổi tác, giới tính, mức độ thu nhập hay kinh nghiệm làm việc, mà chỉ cần xem xét sao 
việc đào tạo đó có thật sự cần thiết cho công việc hay không

Mặc dù còn có những thiếu sót nhất định, nhưng kết quả của nghiên cứu này cũng đã đóng góp một phần nào đó vào lý thuyết chuyển giao đào tạo và đã đạt được mục tiêu đề ra ban đầu của nghiên cứu:

$>$ Nhận diện bốn yếu tố chính của môi trường làm việc có tác động đến chuyển giao đào tạo: cơ hội áp dụng, sự hỗ trợ của đồng nghiệp, sự hỗ trợ của cấp trên và sự sẵn sàng thay đổi.

$>$ Xác định được trong bốn yếu tố của môi trường làm việc có ảnh hưởng đến chuyển giao đào tạo thì yếu tố sự hỗ trợ của đồng nghiệp và sự hỗ trợ của cấp trên có tác động mạnh nhất.

Ngoài ra, đề tài cũng nhằm đưa ra một số đề xuất và hàm ý quản trị đối với các nhà lãnh đạo, các nhà hoạch định và phát triển nguồn nhân lực cho công ty. Qua đó, sẽ giúp cho công ty có cái nhìn thật sâu sắc đến vấn đề chuyển giao đào tạo nhằm xây dựng được đội ngũ nhân viên tinh thông nghề nghiệp.

\subsection{Hàm ý quản trị}

Nhân sự vốn là đề tài được nhiều nghiên cứu chú tâm tìm hiểu với mục đích cuối cùng là làm cách nào để tối ưu hóa nguồn nhân lực và nâng cao trình độ của nhân viên. Một công ty có được nguồn nhân lực chất lượng thông qua tuyển mộ và đào tạo. Kết quả của công ty gắn liền với chất lượng nguồn nhân lực. Đào tạo là một phương pháp giúp chất lượng nguồn nhân lực của công ty ngày càng tăng nhưng để đạt được kết quả tối ưu thì cần có được sự chuyển giao đào tạo. Sự chuyển giao đào tạo có được hay không là do ảnh hưởng của nhiều nhóm yếu tố như: môi trường làm việc, chương trình đào tạo, tính cách nhân viên... trong đó, nhóm yếu tố môi trường được xem như là có tầm ảnh hưởng hàng đầu. Môi trường làm việc gồm có bốn yếu tố chính: cơ hội áp dụng, sự hỗ trợ của đồng nghiệp, sự hỗ trợ cấp trên và sự sẵn sàng thay đổi. Theo như nhận xét chủ quan của tác giả thì hiện tại vấn đề chuyển giao đào tạo ít được chú ý tại Thành phố Hồ Chí Minh nói riêng và tại Việt Nam nói chung. Các công ty thông thường đánh giá nhân viên dựa vào nhu cầu công việc để xem xét đưa đi đào tạo nhưng vấn đề là hiệu quả của việc đào tạo đó như thế nào thì dường như xem nhẹ. Qua kết quả của nghiên cứu "Chuyển giao đào tạo - Tầm quan trọng của môi trường làm việc", thì các bên có liên quan như bộ phận nhân sự, giám sát/quản lý nên tạo cơ hội để cho nhân viên áp dụng được những gì đã được đào tạo vào trong công việc.

> Đối với bộ phận nhân sự: đánh giá kỹ càng nhu cầu của nhân viên trước khi cho tham gia một khóa học đào tạo. Hỗ trợ nhân viên trước, trong và sau khi được đào tạo: tạo điều kiện để nhân viên tham gia khóa đào tạo, thông báo các chương trình đào tạo trước cho nhân viên để họ chuẩn bị tinh thần cũng như về thời gian và công việc; Sử dụng mô hình Kirkpatrick bốn cấp độ để đánh giá hiệu quả đào tạo của từng nhân viên mà kịp thời điều chỉnh theo hướng tích cực để cho chuyển giao đào tạo xảy ra ở mức độ cao nhất.

$>$ Đối với cấp trên/giám sát: chủ động trao đổi với nhân viên, xem xét những khó khăn hay những vấn đề mà nhân viên muốn áp dụng; đặt mục tiêu sau mỗi chương trình đào tạo để cho nhân viên tích cực áp dụng. Bên cạnh đó, cấp trên nên khen ngợi động viên để nhân viên tích cực sử dụng kiến thức đã được đào tạo.

$>$ Đối với đồng nghiệp: Là những người làm chung với nhau trong nhóm làm việc nên để đạt hiệu quả trong công việc thì đồng nghiệp của người được đào tạo cần có thái độ hợp tác, hỗ trợ sự chuyển giao những kiến thức mới đã được lĩnh hội thông qua chương trình đào tạo. Như vậy vừa có lợi cho công việc chung mà những đồng nghiệp này cũng được thụ hưởng lợi ích do được chia sẻ những gì mà người khác được đào tạo. 


\section{TÀI LIỆU THAM KHẢO}

Baldwin, T. T. and Ford, K.J., (1988). Transfer of training: A review and directions for future research. Personnel Psychology, 41, 1, 63-105.

Devos, C., Dumay, X., Bonami, M., Bates, R., \& Holton, E. (2007). The Learning Transfer System Inventory (LTSI) translated into French: Internal structure and predictive validity. International Journal of Training and Development, 11(3), 181-199.

Edwin B. Flippo, Personnel Management, Sixth Edition, McGraw-Hill, New York, 1984.

Gielen, E. (1996, February). Transfer of training in a corporate setting: Testing a model. In Academy of Human Resource Development Conference Proceedings (pp. 434-441).

Foxon, M. (1993). A Process Approach To The Transfer of Training Part 1: The Impact of Motivational And Superior Support on Transfer Maintenance Training And Development Journal. 36(10). pp. 75-78.

Kirckpatrick, DL (1994). Evaluating training programs. The four levels. Berrett- Koehler, San Francisco.

Lim, D. H., \& Johnson, S. D. (2002). Trainee perceptions of factors that influence learning transfer. International journal of training and development, 6, 36-48.

Yamnill, S., \& McLean, G. N. (2001). Theories supporting transfer of training. Human resource development quarterly, 12(2), 195-208. 